\title{
MICROSTRUCTURAL STUDIES OF SPUTTER-DEPOSITED TIN CERAMIC FILMS
}

\author{
A.R. PELTON, B.W. DABROWSKI and L.P. LEHMAN \\ Department of Materials Science and Engineering, University of Notre Dame, Notre Dame, Indiana 46556, USA \\ C. ERNSBERGER \\ CTS Corporation, Elkhart, Indiana 46514, USA
}

\author{
A.E. MILLER \\ Department of Materials Science and Engineering, University of Notre Dame, Notre Dame, Indiana 46556, USA \\ and
}

\section{J.F. MANSFIELD}

University of Michigan Electron Microbeam Analysis Laboratory, Ann Arbor, Michigan 48109-2143, USA

Received at Editorial Office October-November 1988; presented at Conference May 1988

\begin{abstract}
A transmission electron microscopy study was undertaken to characterize the microstructures of TiN thin films that were sputter-deposited onto $\mathrm{Cu}-\mathrm{Be}$, alumina and glass substrates. There were no substantial microstructural differences observed among the films; the average grain size was $150 \mathrm{~nm}$ and the grains were surrounded by growth defect voids which are characteristic of Type-T microstructures. For comparison, the microstructure of TiN that was directly deposited onto carbon-coated TEM grids was also characterized. These films also contained grain-boundary voids, although the average grain size was only $15 \mathrm{~nm}$. A prominent feature of the films deposited on the three substrates was the presence of intragranular microcracks which were usually associated with grain-boundary cusps or facets. These cusps were observed to lie on $\{220\}$ planes with arrays of microcracks emanating from them with a periodicity of approximately $10 \mathrm{~nm}$. It was speculated that due to the extremely high residual stress in these films, these cusps act as stress raisers that promote the formation of the microcracks.
\end{abstract}

\section{Introduction}

Titanium nitride is finding increased use as hard coatings for a wide range of applications including wear-resistant layers on cutting tools, and diffusion barriers and electrical contacts in the microelectronics industry. The commercial importance of this ceramic alloy stems not only from its excellent properties, but also because TiN can be readily processed with modern thin-film technologies. It has been recently recognized, however, that rather large values of internal stresses $(\sim 6$ $\mathrm{GPa}$ ) can be generated in the as-deposited thin films depending on the exact processing condi- tions [1-3]. Rickerby pointed out that there are two components to these internal, or residual, stresses: intrinsic stress and thermal stress [2]. The thermal stresses arise from the differences in the thermal expansion coefficients between the film and substrate, and the intrinsic stresses are due to the growth process which generates structural defects. Obviously, these residual stresses could be detrimental to the performance of the engineered structure and could lead, for example, to spallation of the hard coating or open circuits in microelectronic applications.

Despite the commercial importance of these films and the large volume of scientific literature

0304-3991/89/\$03.50 @ Elsevier Science Publishers B.V.

(North-Holland Physics Publishing Division) 
available, there is a dearth of understanding of the relationships among physical properties, processing parameters and microstructure. In particular, although there have been several investigations with X-ray diffraction and scanning electron microscopy (see, for example, papers in ref. [4]), there have been relatively few transmission electron microscopy investigations of TiN. In order to place the present work in proper context, however, it is worthwhile to review two of the more recent TEM studies of stoichiometric TiN. Hibbs et al. [5] investigated the microstructure of TiN films grown by reactive magnetron sputter-deposition at $1.9 \mathrm{~kW}$ power onto stainless steel substrates at temperatures between 200 and $650^{\circ} \mathrm{C}$. In all films except those deposited at $650^{\circ} \mathrm{C}$ they observed voids and small cracks in and surrounding the grains. As part of this study, they also investigated the effects of substrate bias on the grain size and morphology. They found that the average grain size of the film with no substrate bias was approximately twice as large, approximately $100 \mathrm{~nm}$ compared with $50 \mathrm{~nm}$, as the film grown at -300 $\mathrm{V}$ bias. In another study by the same group [6], it was found that TiN films that were reactively sputtered at $1.9 \mathrm{~kW}$ power contained small, equiaxed grains with $50 \mathrm{~nm}$ grain diameters that were surrounded by $500 \mathrm{~nm}$ square-shaped grains. They observed many intergranular voids as well as "voids with a fingerlike structure" that penetrated the interior of the grains.

The purpose of this paper is to present some preliminary findings from a TEM microstructural analysis of defects in sputtered thin films of stoichiometric TiN and to correlate these results with recent studies of the state of residual stress in the as-deposited films.

\section{Experimental procedures}

\subsection{Thin film deposition}

Stoichiometric TiN films were produced by reactive DC sputtering in a UHV system with a base pressure of $4 \times 10^{-9}$ Torr. The three types of substrates used in the present investigation were a $\mathrm{Cu}-\mathrm{Be}$ alloy, common soda-lime glass microscope slides and electronic grade alumina. The glass and alumina substrates were preheated to 250 and $300^{\circ} \mathrm{C}$, respectively, and sputter-etched with RF power of $250 \mathrm{~W}$ in $5 \mathrm{mT}$ Torr of ultra-high purity argon. The thin films were sputtered at $5.5 \mathrm{~kW}$ and 4 inch cathode-to-substrate distance at a pressure of 4 mTorr. A DC bias of $-100 \mathrm{~V}$ was additionally applied to the substrates during deposition; the $\mathrm{Cu}-\mathrm{Be}$ alloy was biased continously throughout the deposition, whereas the nonconducting substrates could be biased only after an initial layer of TiN was deposited. These conditions allowed a deposition rate of approximately $210 \mathrm{~nm} / \mathrm{min}$ and resultant film thicknesses of approximately $2 \mu \mathrm{m}$. More specific details of the sputtering conditions may be found in refs. $[1,7]$.

Additional thin films were deposited under similar conditions (without DC biasing) on carboncoated $3 \mathrm{~mm}$ diameter TEM grids. The thickness of these films was approximately $80 \mathrm{~nm}$.

\subsection{TEM sample preparation}

Through-section TEM specimens were obtained by cutting small pieces $(-5 \mathrm{~mm} \times 5 \mathrm{~mm})$ of the coated substrates with a low-speed diamond saw perpendicular to the film/substrate interface. These pieces were then mounted with a low-melting wax with the film side down onto a stainless steel cylinder so that the substrate could be cut parallel to the interface. The substrate thickness was subsequently reduced from $150 \mu \mathrm{m}$ to approximately $10 \mu \mathrm{m}$ by mechanically polishing with a series of diamond pastes in a specially modified Minimet polisher. After washing repeatedly in acetone and methanol to remove the wax, these samples were then epoxied between oval copper washers for mechanical stability.

Due to the high residual stresses in the as-deposited thin films (as will be discussed below), the samples were ion-thinned to electron transparency as follows: The samples were ion-milled with two guns at $4 \mathrm{kV} \mathrm{Ar}^{+}$and $40 \mu \mathrm{A}$ beam current with an incident angle of $30^{\circ}$ for approximately one hour. Additional thinning of the substrate was done by ion-milling with one gun until perforation. Final thinning was achieved with dual-beam-milling at an angle of $20^{\circ}$ and at a reduced beam current. It is estimated that this procedure produced thin regions of the TiN approximately midway through 
the film thickness. Limited cross-section films were also studied; the results from this investigation will be discussed in a forthcoming publication.

A JEOL $100 \mathrm{C}$ TEM-STEM operated at $120 \mathrm{kV}$ was used to characterize the films. One sample was additionally characterized with a JEOL $2000 \mathrm{FX}$ at $200 \mathrm{kV}$ at the University of Michigan Electron Microbeam Analysis Laboratory.
Through-focus bright-field images were produced to accentuate grain-boundary voids and microcracks $[8]$.

\section{Experimental results}

Thin films of TiN were deposited onto carboncoated TEM grids in order to investigate the grain

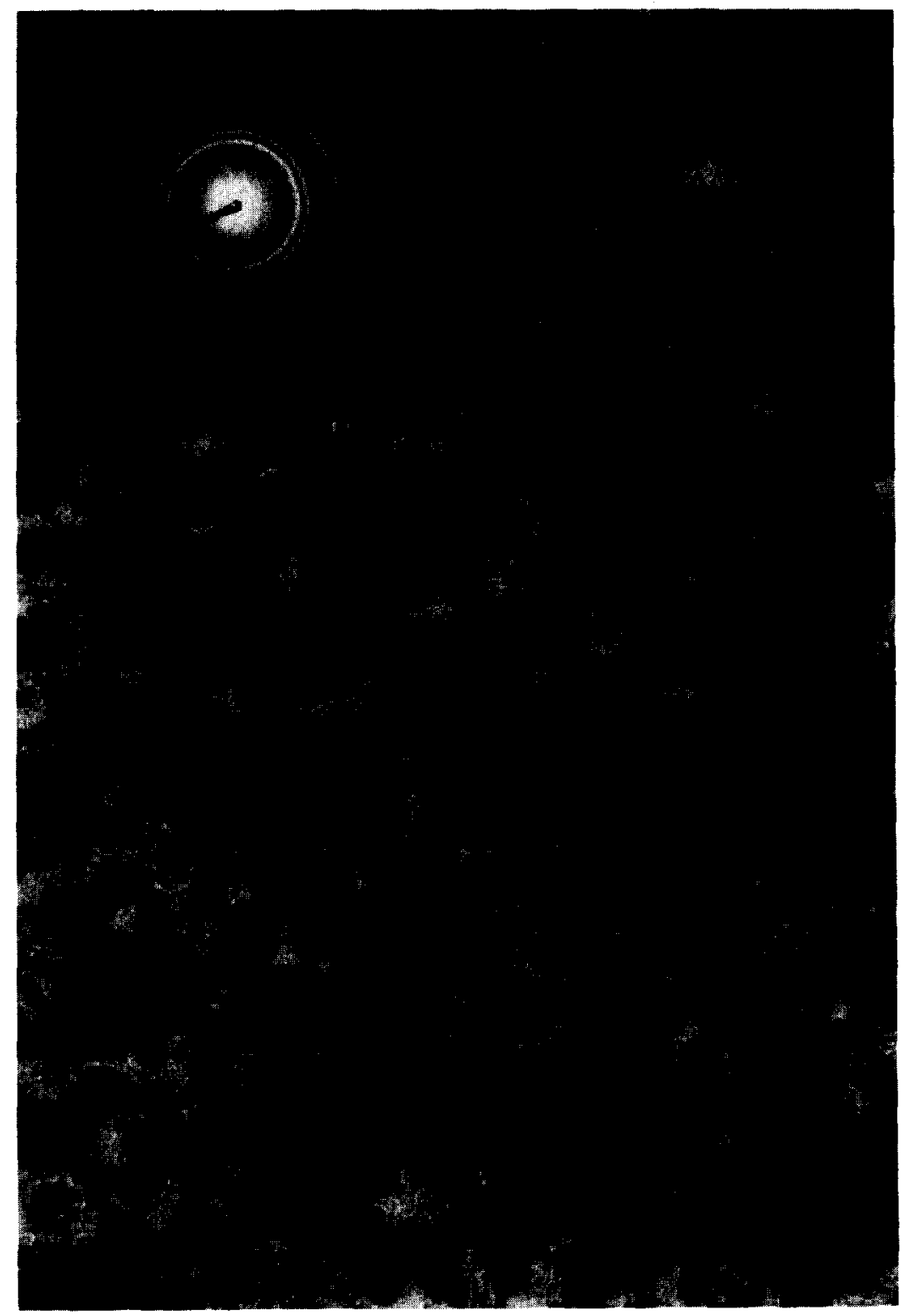

Fig. 1. Underfocussed bright-field micrograph and selected area diffraction pattern of TiN that was deposited directly on a carbon-coated TEM grid. Bar equals $100 \mathrm{~nm}$ 
morphology and other microstructural features in the incipient layers. Fig. 1 shows an under-focussed bright-field image and corresponding selected area diffraction pattern from such a film. The average grain size is approximately $15 \mathrm{~nm}$ with a range of
5 to $25 \mathrm{~nm}$. At this magnification it is difficult to determine if there are any microstructural defects such as dislocations or small-angle boundaries within the grains. It is apparent, however, that the grains are surrounded by grain-boundary voids.
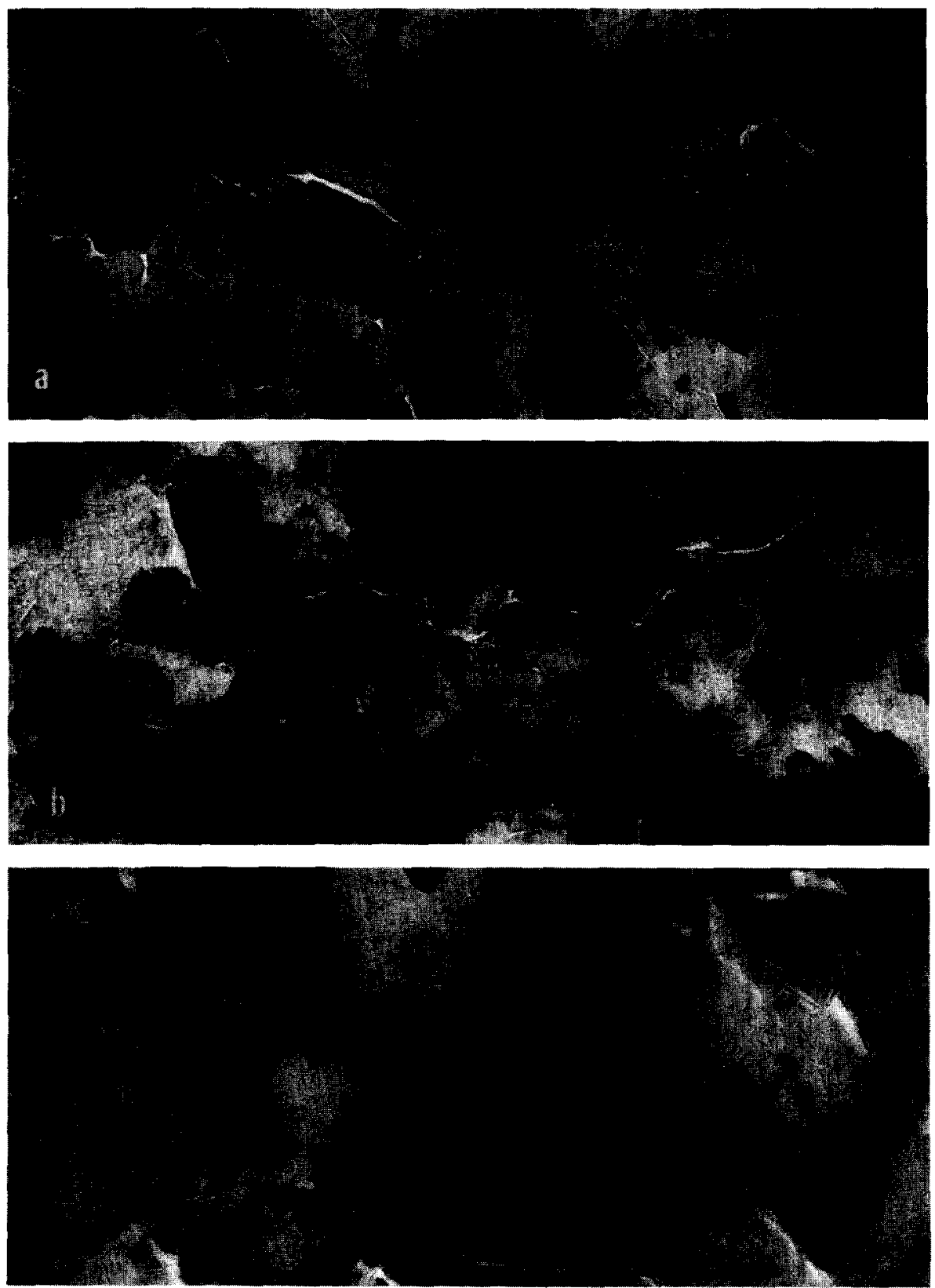

Fig. 2. Bright-field images of TiN deposited onto $\mathrm{Cu}-\mathrm{Be}$ (a), alumina (b), and glass (c) substrates. Note the grain-boundary voids (large, open arrows) and the intragranular microcracks (small arrows). Bar equals $100 \mathrm{~nm}$. 
This voided boundary structure is common in thin films that are sputtered with relatively low homologous temperatures and is characteristic of so-called Zone T microstructures [9]. The diffraction pattern from this region was fully indexed according to the $\mathrm{NaCl}$ crystal structure with a lattice parameter of $0.424 \mathrm{~nm}$. No extra reflections from any second phases were observed in the SADP, which is consistent with the quantitative chemical analysis from Auger spectroscopy that indicates that the films were stoichiometric with low values of oxygen [1]. Furthermore, it can be seen that the diffraction pattern does not show any preferred crystallographic texture.

Fig. 2 shows a comparison of the TiN microstructures deposited onto $\mathrm{Cu}-\mathrm{Be}$ (fig. $2 \mathrm{a}$ ), alumina (fig. 2b) and glass (fig. 2c) substrates. There does not appear to be any fundamental differences in the microstructures of these three samples. At this location in the films (approximately $1 \mu \mathrm{m}$ from the substrate/film interface) the average grain size of these films is about $150 \mathrm{~nm}$ with a wide variation in size. It should be noted that exact grain size measurements were extremely difficult to make because of the variability in diffraction contrast across the field of view. Furthermore, these bright-field images were produced at non-Gaussian objective focus to accentuate the visibility of the omnipresent grain-boundary voids, some of which are marked with the large, open arrows in fig. 2. Complementary focussed images of these samples were obtained under two-beam brightfield and weak-beam dark-field conditions to enhance visibility of possible dislocation structures.
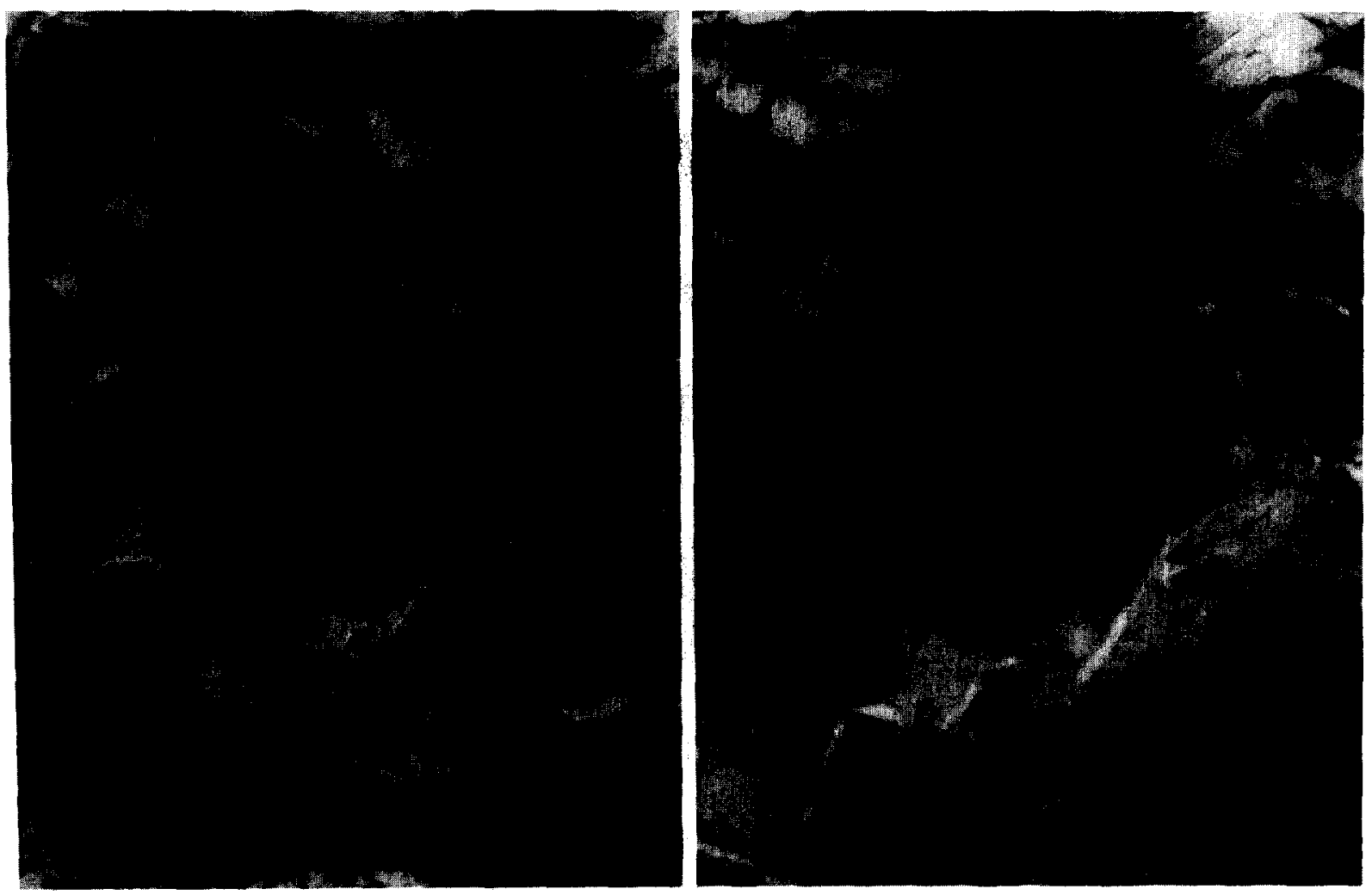

Fig. 3. Low-magnification (a) image of TiN deposited on glass. Note the grain-boundary voids and "cusps" and the associated microcracks. The boxed region is shown at higher magnification in (b). The arrows illustrate microcracks that are associated with cusps. Bars equal $100 \mathrm{~nm}$. 
Even under proper imaging contrast, clear images of dislocations were rarely observed in any of the samples.

In addition to the grain-boundary void structures, a network of intragranular microcracks is observed within the majority of the grains of these samples. Some of these microcracks are arrowed in the three micrographs in fig. 2. These microcracks were characterized according to size, shape, distributions and crystallographic orientations in order to understand their possible origin. In many areas, the microcracks traverse the entire grain and appear to lie along specific crystallographic planes. In other regions, the cracks are much shorter and wavier as seen near the small arrow in fig. 2c. Another common feature of the microcracks is that they appear to originate at the grain boundaries and are usually associated with some prominent feature of the grains. For example, many of the grain surfaces are faceted or cusped and the microcracks appear to originate at these morphological features as seen in several areas in fig. 2; additional examples of the relationship between cusps and microcracks will be illustrated in subsequent figures.

Since there were few discernible differences in the microstructures of the TiN films obtained from the three substrates, we will further illustrate our observations of the microcracks with examples from the glass substrates. Fig. 3a shows a lowmagnification image in the overfocussed condition in which the grain-boundary voids are visible as black lines. Many of these boundary voids or "macrocracks" are branched into the grains and appear to be associated with the intragranular cracks. The boxed region in this figure is shown at higher magnification in fig. 3b. This particular region is quite rich with microcracks which tend to

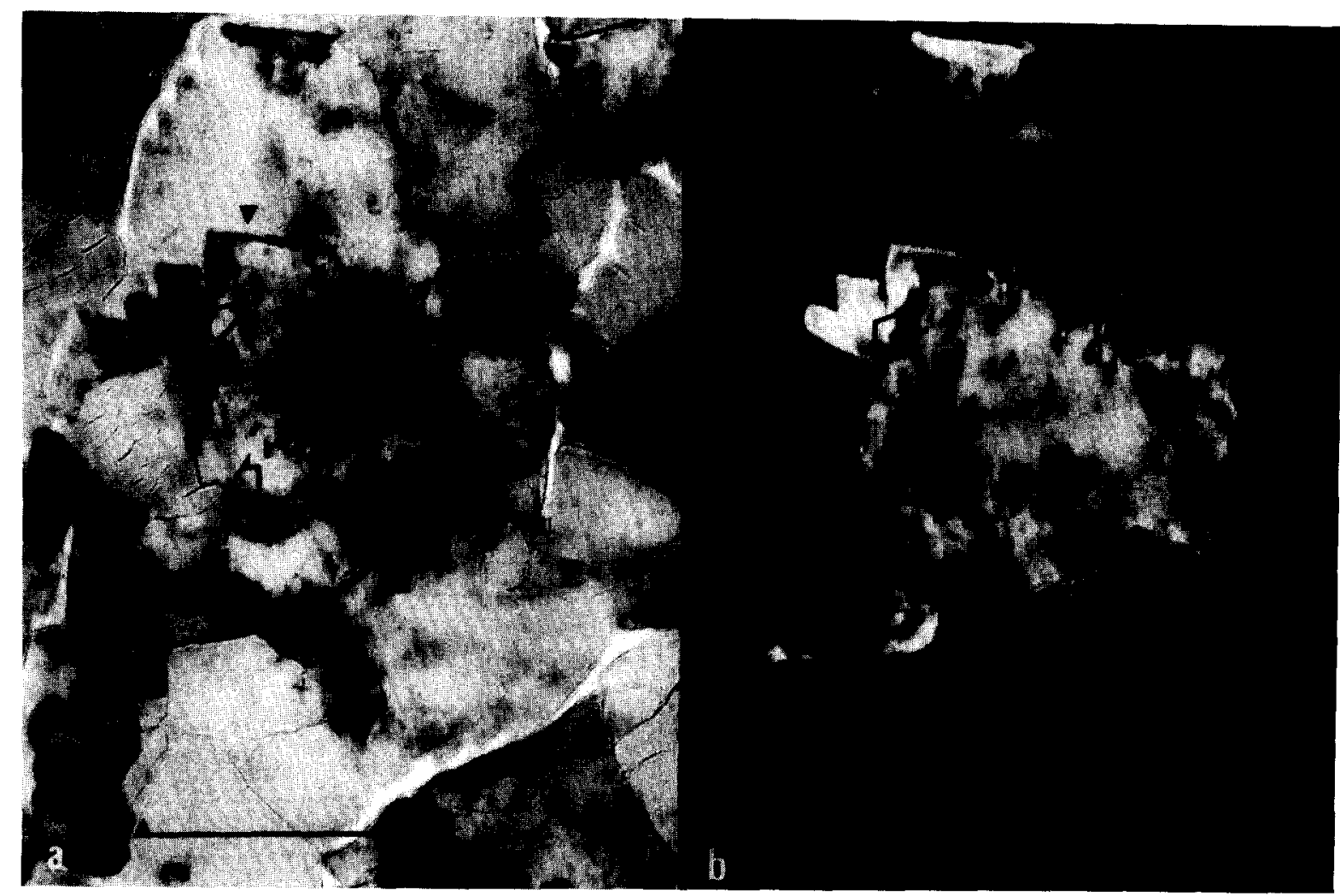

Fig. 4. Bright-field (a) and dark-field (b) images that show extreme grain-boundary cusps (large arrows), microcracks and small-angle grain boundaries (small arrows). Bar equals $100 \mathrm{~nm}$. 
originate at the grain boundary voids; note, for example, the microcrack arrays that are marked with arrows. In particular, the region near the upper arrow in this figure illustrates the faceted nature of the grains and the obvious association of the microcracks. An additional example of the extreme grain faceting and associated microcracks in these TiN films is marked with large open arrows in the bright-field, dark-field pair in fig. 4 . These micrographs also show small-angle grain boundaries (small arrows) that are occasionally observed in these films. Furthermore, it is ap-
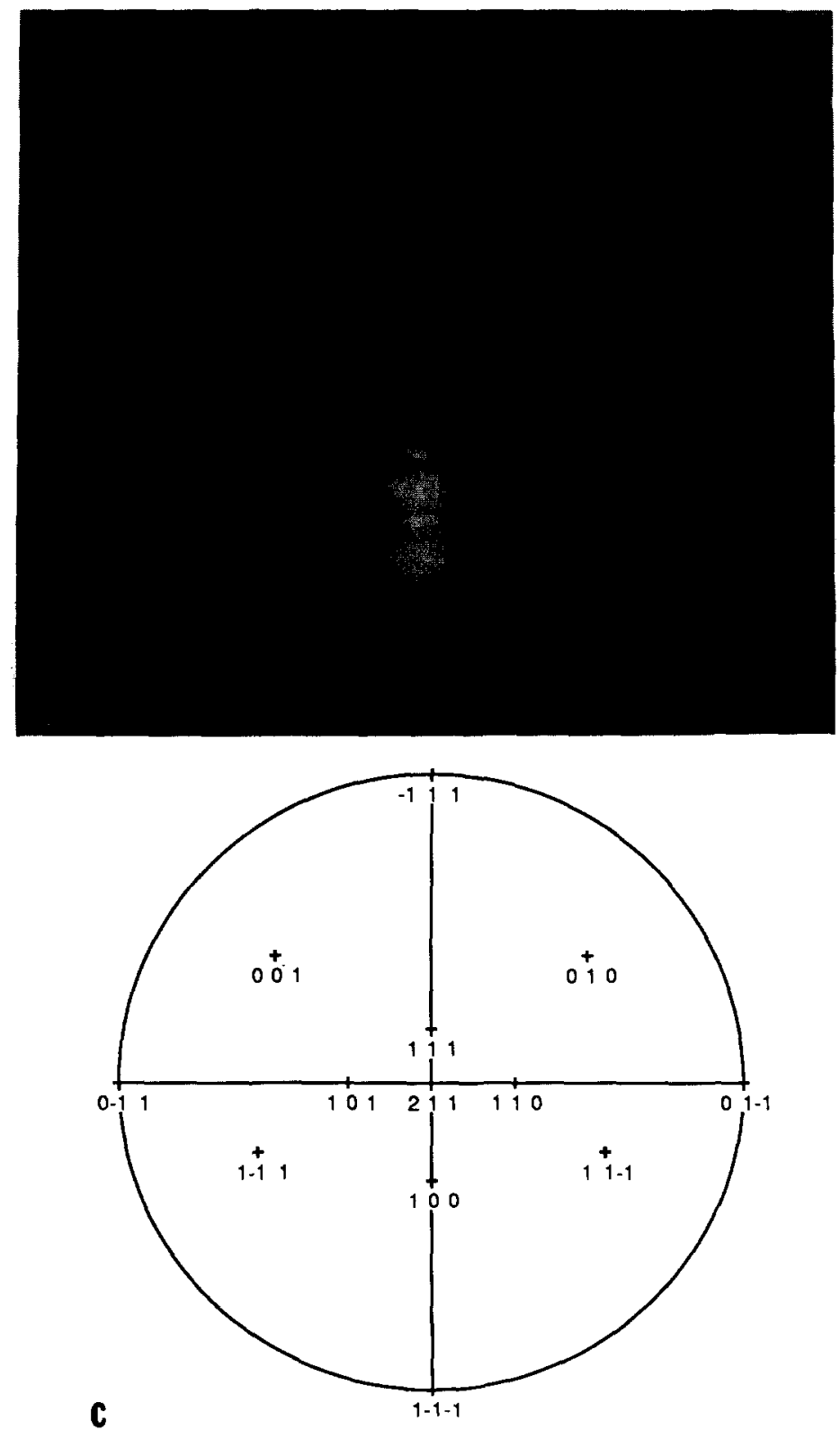

Fig. 5. Bright-field image (a) of grain-boundary cusps, corresponding [211] microdiffraction pattern (b), and stereographic projection (c). Bar equals $50 \mathrm{~nm}$. 
parent from these micrographs that microcracks are only visible in those grains with kinematic imaging conditions. The visibility of the cracks was also observed to be affected by small degrees of specimen tilt, which indicates that there may be a component of strain contrast in the images.

One of the goals of this investigation was to relate the microcracks to the crystallography of the TiN grains. However, it was not always possible to obtain unambiguous determinations of the crack plane; only limited numbers of images and diffraction patterns were able to be analyzed fully. One example of such an analysis is demonstrated in fig. 5, which shows a bright-field image, corresponding [211] microdiffraction pattern and [211] stereographic projection [10]. The underfocussed image in fig. $5 \mathrm{a}$ is from an approximately $125 \mathrm{~nm}$ diameter grain with a number of cusps along the grain boundary. Several images were obtained from this grain under various imaging conditions around the minor stereographic triangle [111], [110], and [211]. From the highly kinematical images in this series it was observed that there were one or more microcracks that ran into the grain from, and parallel to, each of these cusps. It is clear from the microdiffraction pattern in fig. $5 b$ that the cusps lie along the [1111] direction. An analysis of the [211] stereographic projection in fig. $5 \mathrm{c}$ indicates that the only low-index planes that lie on the (111) trace are $(0 \overline{1} 1),(101)$ and (110). Comparison with images from the [110] and [111] zones suggest that the cracks lie on (0ī1) planes. Additional results were obtained from other regions that also suggest a predominant $\{110\}$ fracture plane for many of the microcracks.

The microcrack spacing was measured on nearly thirty micrographs from all three substrates, and the average spacing was determined to be approximately $10 \mathrm{~nm}$. The average spacing was observed in all of the various crack arrays including the long, straight cracks and the short, wavy cracks.

\section{Discussion}

The microstructural results from the TiN films that were deposited onto the carbon-coated grids, fig. 1, played a key role in the understanding of the microstructures observed in the other samples reported above. First, the observation of the extremely small grain size $(\sim 15 \mathrm{~nm})$ in these films is consistent with our findings of cross-sectional TiN films that there is a substantial change in the grain size from the film/substrate interface to the free surface. This change in grain size was also observed in sputter-deposited films of $\mathrm{NbN}$ by Ho et al. [11]. From cross-section TEM samples they found that the average grain size increased from $10 \mathrm{~nm}$ at the interface to over $120 \mathrm{~nm}$ at a distance of $2 \mu \mathrm{m}$ from the interface. Second, the presence of grain-boundary voids in the films on the carbon-coated grids established that there was not a substantial difference in the morphology of the TiN grains deposited on the various substrates reported in this study. Furthermore, these results indicate that the energy of the deposited films (in this case, $5.5 \mathrm{~kW}$ ) dictates the amount of grainboundary defects.

The microstructural observations of the thin films deposited onto the $\mathrm{Cu}-\mathrm{Be}$, alumina and glass substrates will be discussed in terms of previously reported findings. In particular we will interpret the present results in conjunction with a companion publication by Ernsberger et al. [1]. In the Ernsberger paper, the residual stress of the TiN thin films was measured as a function of temperature and film thickness by means of in-situ cantilever beam-bending experiments. They observed that sputtered thin layers $(\sim 0.2 \mu \mathrm{m})$ on quartz beams resulted in pronounced beam deflections. These deflections were converted into values of the modulus of elasticity $(595 \pm 131 \mathrm{GPa})$ and residual stress (4 to $6 \mathrm{GPa}$ ) following the analysis of $\mathrm{Hu}$ et al. [12]. Since the beam was deflected down, it is obvious that there is a substantial compressive component of the total internal stress. Furthermore, it was determined that the thermally induced stresses in the films deposited onto quartz amounted to approximately $500 \mathrm{MPa}$ per $100^{\circ} \mathrm{C}$.

$\mathrm{X}$-ray stress measurements by Ernsberger et al. [1] further indicate that there is a stress gradient over the thickness of the film deposited onto glass slides. This gradient ranged from a tensile stress in the thin layer of material at the film/substrate interface to a complex mixture of compressive and shear stresses in the body of the film. These results 
are contrary to the earlier findings of Rickerby [2], who only considered a compressive in-plane stress and a tensile perpendicular stress in sputtered TiN films.

An estimate of the amount of strain in these films may be obtained from the values of the modulus of elasticity and the amount of residual stress; strains of $6 \mathrm{GPa}: 595 \mathrm{GPa}$ or approximately $1 \%$ strain can be obtained. Although precise values of the yield strength and tensile strength of TiN are not known, it is certain that the state of stress measured in these films must be near the elastic limit. Accordingly, Rickerby [2] considered the various mechanisms by which the films may relieve a portion of the residual stresses and strains:

(1) Plastic deformation in the substrate.

(2) Plastic flow of the deposited film: (a) within the volume of the grain; (b) at the grain boundaries; (c) at free surfaces.

(3) Fracture or buckling of the film

From this senario, it is apparent that the residual stresses in the present films are relieved primarily by the third mechanism, namely, cracking. It is also tempting to re-evaluate the microstructural results from Hibbs et al. [5,6] in the light of the present findings. As summarized in the Introduction, Hibbs and co-workers observed small cracks that they interpreted as growth defects in many of their films. However, it is possible that their deposition parameters were sufficient to generate moderately high residual stress levels, especially in those films in which the substrates were biased. Since they also observed dislocations under some of their processing conditions, it is possible that the residual stresses in their films were accommodated by a combination of film yielding and fracture.

Furthermore, many of the morphological features of the grains that were observed by Hibbs et al. were found in the present work. In particular, their "fingerlike" voids [6] resemble our cusped or faceted grains shown in figs. 2-5. Although these cusps are certainly due to growth defects, they apparently also play a role in the stress-relieving mechanism of the grains. It is possible that under the high compressive and shear stresses these cusped regions are local stress raisers. As such, microcracks originate at these growth defects. In addition, the measured $10 \mathrm{~nm}$ crack spacings could reflect the periodicity of the cusps.

The $(0 \overline{2} 2)$ fracture planes observed in fig. 5 are also consistent with the results of Hibbs et al. [5]. Their "fingerlike" voids were found to grow in $\langle\overline{1} 11\rangle$ directions in $\{211\}$ planes, which suggests $\{0 \overline{2} 2\}$-type fracture planes. Since TiN has a B1 crystal structure based on the FCC Bravais lattice, it would be expected that the primary slip and shear planes would be $\{111\}$ or $\{200\}$. However, it is also well known that TiN has a complex mixed bonding with components of covalent, ionic and metallic bonds [13]. Due to this complex bonding, it is likely that other slip and fracture systems could be activated.

Finally, it is interesting to speculate on the importance of atomic defects (such as vacancies, interstitials, trapped gas atoms, and ions on the wrong sublattices) that are inherent in sputtered thin films and enhanced by substrate bias with respect to the fracture mechanisms. In a recent paper, Perry [14] discussed the influence of these point defects on the physical properties of TiN and related ceramic films. He showed that the presence of these defects has a pronounced effect on the density, lattice parameter, reflectance and color of the films. In light of the present observations, it is also highly probable that the point defects could cluster and allow a preferred fracture path under conditions of high internal stress. On the other hand, Ho et al. [11] argued that the microcracks in NbN may not exist in the as-deposited films. They suggested that the presence of these atomic defects could lead to preferential cracking during ion-thinning TEM specimens of $\mathrm{NbN}$. Although this mechanism cannot be ruled out entirely, based on the residual stress measurements discussed above, it is more probable that microcracks exist in the as-deposited films, and that the presence of the point defects may influence the fracture of the films.

\section{Conclusions}

We observed the microstructures of sputter-deposited thin films of $\mathrm{TiN}$ on $\mathrm{Cu}-\mathrm{Be}$, alumina, and 
glass substrates. There were no discernible differences in the microstructures of the films from the three substrates; the grain size was approximately $150 \mathrm{~nm}$ and the grains were surrounded by voids. For comparison, the microstructure of TiN deposited directly on carbon-coated TEM grids under similar conditions was also characterized. Grain boundary voids were also observed in these films, although the grain size was only $15 \mathrm{~nm}$.

A prominent feature of the films deposited on the three substrates was intragranular microcracks which were usually associated with grain boundary cusps or facets. These cusps were observed to lie on $\{220\}$ planes with arrays of microcracks emanating from them. It was speculated that due to the extremely high residual stress in these films (4-6 GPa [1]) these cusps act as stress raisers that promote the formation of the microcracks. It was additionally observed that many of the microcracks appear to lie on $\{220\}$ fracture planes, which is consistent with previous results $[5,6]$.

It was further speculated that the presence of high densities of point defects could influence the mechanical integrity of the sputtered TiN films, and could assist in the fracture mechanism.

\section{Acknowledgements}

The authors gratefully acknowledge the continuing support of CTS Corporation and the Indiana Corporation for Science and Technology. A.R.P. and B.W.D. additionally acknowledge a grant from the Jesse H. Jones Research Fund at the University of Notre Dame. We appreciate the efforts of Tom Smith who helped in the sample preparation and was the source of much useful information. Stimulating discussions with Dr. Anthony J. Perry were extremely helpful. Finally, the micrographs in fig. 5 were obtained on the JEOL 2000FX at the University of Michigan Electron Microbeam Analysis Laboratory.

\section{References}

[1] C. Ernsberger, A.J. Perry, L.P. Lehman, A.E. Miller, A.R. Pelton, and B.W. Dabrowski, Surface and Coatings Technol. 36 (1988) 605.

[2] D.S. Rickerby, J. Vacuum Sci. Technol. A4 (1986) 2809.

[3] A.J. Perry and L. Chollet, J. Vacuum Sci. Technol. A4 (1986) 2801.

[4] Proc. 13th Intern. Conf. on Metallurgical Coatings, San Diego, CA, 1986, Ed. R.C. Krutenat [J. Vacuum Sci. Technol. A4, No. 6 (1986)]

[5] M.K. Hibbs, B.O. Johansson, J.-E. Sundgren and U. Helmersson, Thin Solid Films 122 (1984) 115.

[6] M.K. Hibbs, J.-E. Sundgren, B.E. Jacobson and B.O. Johansson, Thin Solid Films 107 (1984) 149.

[7] C. Ernsberger, J. Nickerson, T. Smith, A.E. Miller and D. Banks, J. Vacuum Sci. Technol. A4 (1986) 2784.

[8] D.R. Clarke, J. Am. Ceram. Soc. 63 (1979) 104.

[9] J.A. Thorton, Ann. Rev. Mater. Sci. 7 (1977) 239.

[10] T.H. Kosel, Crystal Software, Stereographic Projection (1987).

[11] H.L. Ho, R.T. Kampwirth, K.E. Gray, D.W. Capone II, L.S. Chumbley and M. Meshii, Ultramicroscopy 22 (1987) 297.

[12] C.K. Hu, D. Gupta and P.S. Ho, in: Proc. Intern. IEEE VLSI Multilevel Interconnect Conf., 1985, p. 185.

[13] J.-E. Sundgren, B.O. Johansson, A. Rockett, S.A. Barnett and J.E. Greene, AIP Conf. Proc. (1986) 95.

[14] A.J. Perry, J. Vacuum Sci. Technol. 6 (1988) 2140. 